\title{
Context dependent role of p53 up-regulated modulator of apoptosis (puma) during liver degeneration and regeneration
}

\begin{abstract}
Hepatocytes are susceptible to injury due to its contribution in xenobiotic metabolism including drugs and alcohol, fatty acid metabolism and enterohepatic circulation of bile acids and hepato tropic viruses. On the other hand regeneration of hepatocytes is well-studied phenomenon essential for maintenance of its volume during chronic liver injury as well as acute restoration of liver volume after resection. During chronic and acute insult of liver apoptosis and necrosis are the most widely documented forms of hepatocyte cell death. Experimental evidence also demonstrates that apoptosis and cell proliferation are closely linked and many proteins that can induce cell death are, in fact, components of the cell division cycle. Apoptosis is crucial for sustain tissue homeostasis and its circumvention is assumed as a hallmark of cancer. Conversely, there are several evidences also support the contribution of pro-apoptotic Bcl-2 family members in development of liver cancer. They also play vital role during liver regeneration induced by partial hepatectomy, a well characterized In vivo model of cell cycle progression. Here we discuss the context dependent role of specifically one $\mathrm{BH} 3$ only group of Bcl-2 family member, Puma during hepatic degeneration and regeneration. Up regulation and down regulation of this specific protein is vital for carcinogenesis as well as hepatic regeneration. The expression levels of Puma contributing to hepatocyte injuries, hepatic proliferation and progression of malignancies are also reflected in the context of potential therapeutic strategies.
\end{abstract}

Volume 2 Issue 3 - 2016

\author{
Subhrajit Biswas \\ Department of Molecular and Cellular Medicine, Institute of \\ Liver and Biliary Sciences, India
}

Correspondence: Subhrajit Biswas, Department of Molecula and Cellular Medicine, Institute of Liver and Biliary Sciences, New Delhi, India, Email subhrajit.biswas9@gmail.com

Received: May 30, 2016 | Published: August 18, 2016

Keywords: hepatocytes, liver apoptosis, lymphomagenesis, tumorigenesis, dietary antigens

Abbreviations: PUMA, p53 up-regulated modulator of apoptosis; HCC, hepatocellular carcinoma; DEN, di ethyl nitrosamine; $\mathrm{PH}$, partial hepatectomy; ROS, reactive oxygen species; MOMP, mitochondrial outer membrane permeabilization; ALD, alcoholic liver diseases; NAFLD, non alcoholic liver diseases comprising fatty liver diseases; NASH, non-alcoholic steatohepatitis; HBV, hepatitis b virus; $\mathrm{HCV}$, hepatitis c virus; HCC, hepatocellular carcinoma; ACLF, acute or chronic liver failure; ROS, reactive oxygen species; ER, endoplasmic reticulum; $\mathrm{PH}$, partial hepatectomy; $\mathrm{KC}$, keratinocyte chemoattractant; MIP-2, macrophage inflammatory protein-2; VEGFR, vegf receptor; PDGFR, pdgf receptor

\section{Introduction}

The group of Bcl-2 family member proteins play a fundamental role in apoptosis and cancer development. ${ }^{1}$ The $\mathrm{BH} 3$ only members Bid, Puma, Bad, Noxa in Bcl-2 family initiate apoptosis in response to a broad range of stimuli and cell types. ${ }^{2}$ Within these initiator proteins, PUMA (P53 Up-Regulated Modulator Of Apoptosis) is a highly conserved apoptosis initiator and was discovered initially as a transcriptional target of $\mathrm{p} 53 .^{3-5}$ Puma is markedly induced in a p53-dependent or independent manner with sense of distinct stimuli such as, DNA damage, oxidative stress and serum deprivation..$^{6-8}$ The protein indirectly dissociates Bax and Bcl-xL and release Bax to set off Mitochondrial Outer Membrane Permeabilization (MOMP) that is associated with its potent pro apoptotic activity. ${ }^{9}$ Puma can also directly associates with Bax to induce apoptosis ${ }^{10}$ and participates in the endoplasmic reticulum associated apoptotic pathway. ${ }^{11,12}$ There were down regulation of Puma has been reported in melanoma and leukaemia, in contrast the increased Puma expression leads to extensive apoptosis in a variety of human cancer cells in vitro as well as xenograft model. ${ }^{13,14}$ Inhibition of Puma with short hairpin RNAs suppressed p53-dependent apoptosis, endorsed the oncogenic transformation of primary murine fibroblasts by E1A/Ras and dramatically steps up El-myc-induced lymphomagenesis. ${ }^{15}$ Loss of Puma enhanced intestinal tumorigenesis induced by carcinogens. ${ }^{16}$ In contrary, two independent studies also demonstrated that Puma dependent apoptosis is required for radiation-induced lymphoma in mice. ${ }^{17,18}$

Here we discuss the contrasting role of Puma in liver regeneration and injury. During liver injury the molecular targets of hepatocyte death in relation to compensatory proliferation have not been fully characterized still yet. There is classical thought that the apoptotic death secures the removal of hepatocyte with compromised genomes and is thought to prevent tumorigenesis. But there are emerging concepts implicate the excessive apoptosis as a keystone in hepatic fibro genesis. The loss of Puma activity can also limit the replicative stress by protecting bone marrow hematopoietic cells from death. ${ }^{18}$ In contrast the Puma mediated apoptosis drives DEN induced mice model of HCC. ${ }^{16}$

\section{Liver degeneration leads to failure of hepatic regeneration}

In our daily life liver cells are continuously exposed to a large number of antigenic loads that includes pathogens, toxins, tumor 
cells and dietary antigens. Hepatic injury or degeneration may cause different aetiologies of diseases including Alcoholic Liver Diseases (ALD), Non Alcoholic Liver Diseases Comprising Fatty Liver Diseases (NAFLD), Non-Alcoholic Steatohepatitis (NASH), autoimmune hepatitis, primary biliary cirrhosis, sclerosing cholangitis etc. Within several years only viral Hepatitis B and C (HBV, HCV) can cause chronic hepatitis which ultimately can progress to cirrhosis and Hepatocellular Carcinoma (HCC). ${ }^{19,20}$ During acute liver insult or in acute on chronic liver failure (ACLF), ${ }^{21}$ the liver micro environments are exposed to instant high levels of pro inflammatory cytokines such as TNF- $\alpha$, IL- $1 \alpha$, INF- $\gamma$, caused by host derived (DAMPs) or pathogen derived (PAMPs) danger signals (E.g: LPS). These cytokines, besides further potentiating the inflammatory response, activate both survival and cell death pathways depending on the exposure and cellular context. On the other hand the activation of Hepatic Stellate Cells (HSCs) and their Trans differentiation into myofibroblast with the secretion of extracellular matrix proteins plays a critical role in fibro genesis. These activated HSCs engulf the apoptotic bodies resulting from hepatocyte death, and produce pro fibrogenic cytokines (such as transforming growth factor- $\beta 1$, TGF- $\beta$ ) and type I collagen..$^{22}$ In the later stages the HSCs also trans differentiate into myofibroblast matrix proteins. Current information suggests that damaged hepatocyte cells release the nucleotides ATP and UTP, which can bind to purinergic G protein coupled P2Y2 receptors the not only present in macrophages but also in the HSCs. ${ }^{22}$ Therefore, emerging concepts implicate excessive hepatocyte death as a keystone in hepatic inflammation and fibro genesis by continuous HSCs activation. Pro and anti apoptotic activities within a particular cell decide whether the balance will tip to one side or the other during liver injury.

The liver is a highly regenerative organ and the predominant $70 \%$ to $80 \%$ hepatocyte cells regulate intermediary metabolism, detoxify endo and xenobiotics, manufacture critical circulating proteins and generate bile acid dependent bile flow. In partial hepatectomy model of mice the $70 \%$ surgical resection of the liver allows the normally quiescent hepatocytes quickly entering the cell cycle to regenerate the original hepatic mass. In rodents this histological restitution of the liver mass is accomplished within 7-10days and in humans this may take about $6-8$ weeks. ${ }^{23}$ During chronic liver injury due to aberrant regeneration with increased expression of TIMPs by resisting the scar resolving function of MMPs, the liver undergoes scarring by inhibiting the proliferation of mature epithelial cells with down regulation of CXCR7 and upregulation of CXCR4. Large number of hepatocyte cells undergo frequently in senescent stage. ${ }^{24}$ But after acute or even repetitive injury, the liver regenerates efficiently ${ }^{25,26}$ recovering from even considerable necrosis caused by toxins or a viral infection. This process requires a coordinated response of immune cells including $\mathrm{T}$ cells, macrophages and eosinophils, mobilization of liver growth factors, matrix remodelling and a rapid but tightly controlled abundance of epithelial cells to sustain the cell cycle and proliferation of hepatocyte. . $^{24,27,28}$

\section{Suppressive mode of Puma is essential for hepatic regeneration}

The balance between the hepatocyte proliferation and cell death is of the highest importance in the regenerative process. ${ }^{29}$ After partial hepatectomy the remnant liver generates an excess of Reactive Oxygen Species $(\mathrm{ROS})^{30}$ where the pro survival Bcl-xL levels increase in response to oxidative stress. ${ }^{31}$ However, pro apoptotic
Bax also increases accordingly. ${ }^{32}$ Even in normal proliferative cells the regulation of Endoplasmic Reticulum (ER) mediated calcium homeostasis by the Bcl-2 family proteins was formerly defined in the context of apoptosis under ER stress, oxidative stress and other adverse conditions. ${ }^{33,34}$ This regulation is not likely only specific to the apoptotic stimulation, but may rather be a constitutive aspect with diverse functional outcomes. Liver regeneration involves both proliferation and apoptosis of hepatocyte. Therefore, the occurrence of rare apoptotic hepatocytes during the early phase of liver regeneration is tricky to describe based on the expression patterns of these Bcl-2 members. ${ }^{35}$

Chen $\mathrm{S}$ et al. ${ }^{36}$ explored the role of Puma and its association with other Bcl-2 family members in liver regeneration by comparing differential expression of Puma with normal livers and hepatitis as well as with hepatoma tissues. To understand the relationship of hepatocyte survival and death of regenerative hepatocyte during liver regeneration, they have measured the Puma mRNA and protein expression in mice model of $70 \%$ Partial Hepatectomy $(\mathrm{PH})$ and found significant decrease of Puma level within 24hour post $\mathrm{PH}$ along with increased Bcl-xL expression without affecting the caspase 3 cleavage (Figure 1) ${ }^{36}$ Similar observation was also found in rats. ${ }^{32}$ Additionally, the expression of Slug protein, the transcriptional repressor of Puma ${ }^{37}$ along with p53, Bax, Bcl-xL increased after PH. So the enforcement of regeneration with $\mathrm{PH}$ concurrently repressed the expression of Puma coupled with Bcl-xL up-regulation, suggesting that the synergetic changes of Puma and Bcl-xL may contribute to suppress apoptosis and resist metabolic overload. But these down regulation could not be explained by the accumulation of ROS,${ }^{30}$ a common stimulus inducing Puma after PH or by the nuclear translocation of $\mathrm{p} 53,{ }^{38}$ also a Puma inducer. Chai group demonstrated the contribution of Puma to liver regeneration was also assessed by manipulating its expression level using adenovirus vectors. They used the adenoviral mediated Puma expression to prevent the physiological down regulation post $\mathrm{PH}$ and showed a decrease in proliferation and an increase in apoptosis in livers after PH, with inflammatory cell infiltration and increased mortality. In addition Puma expression induced potent cytokines Keratinocyte Chemo attractant $(\mathrm{KC})$ and Macrophage inflammatory protein-2 (MIP-2) which is reported to increase in inflammation induced by Fas-mediated hepatocyte death. ${ }^{39}$ These results point out that the increased Puma levels may affect the expression of both pro survival and pro-apoptotic Bcl-2 family partners that influence the hepatocytes likely to die, suggesting that the Puma repression is necessary in the priming and progression phase of liver regeneration. In contrast from this regenerative liver, Puma expression showed an increased trend in human hepatitis, mouse model of carcinogen induced liver injury and hepatoma cells. In fact, the slight increase expression of Puma leads aberrant regeneration inducing apoptosis and highly expressed Puma can cause acute or sudden uncontrolled liver injury with inflammation. ${ }^{36}$ The above collective studies clearly suggest the protective low expression of Puma in hepatocyte during normal proliferation process and liver regeneration (Figure 1)

In the normal adult liver, hepatocytes are either mitotically quiescent stage or divide normally with low expression of Puma. Following partial hepatectomy endothelial growth factor, NF-k $\beta$ mediated proliferation of hepatocyte increase with down regulation of Puma along with up regulation of Bcl-xL to suppress apoptosis. In chronically injured or degenerative liver, the Puma expression is up regulated with increasing inflammatory signals. During HCC progression Puma expression is elevated only in malignant cells but 
not in non malignant hepatocytes. Puma mediated apoptosis and the compensatory proliferation of malignant cells leads to carcinogenesis. Sorafenib treatment inhibits the proliferation of malignant cells as well as induces their apoptotic death, whereas the non-malignant cells are protected from sorafenib induced death due to low expression of Puma.

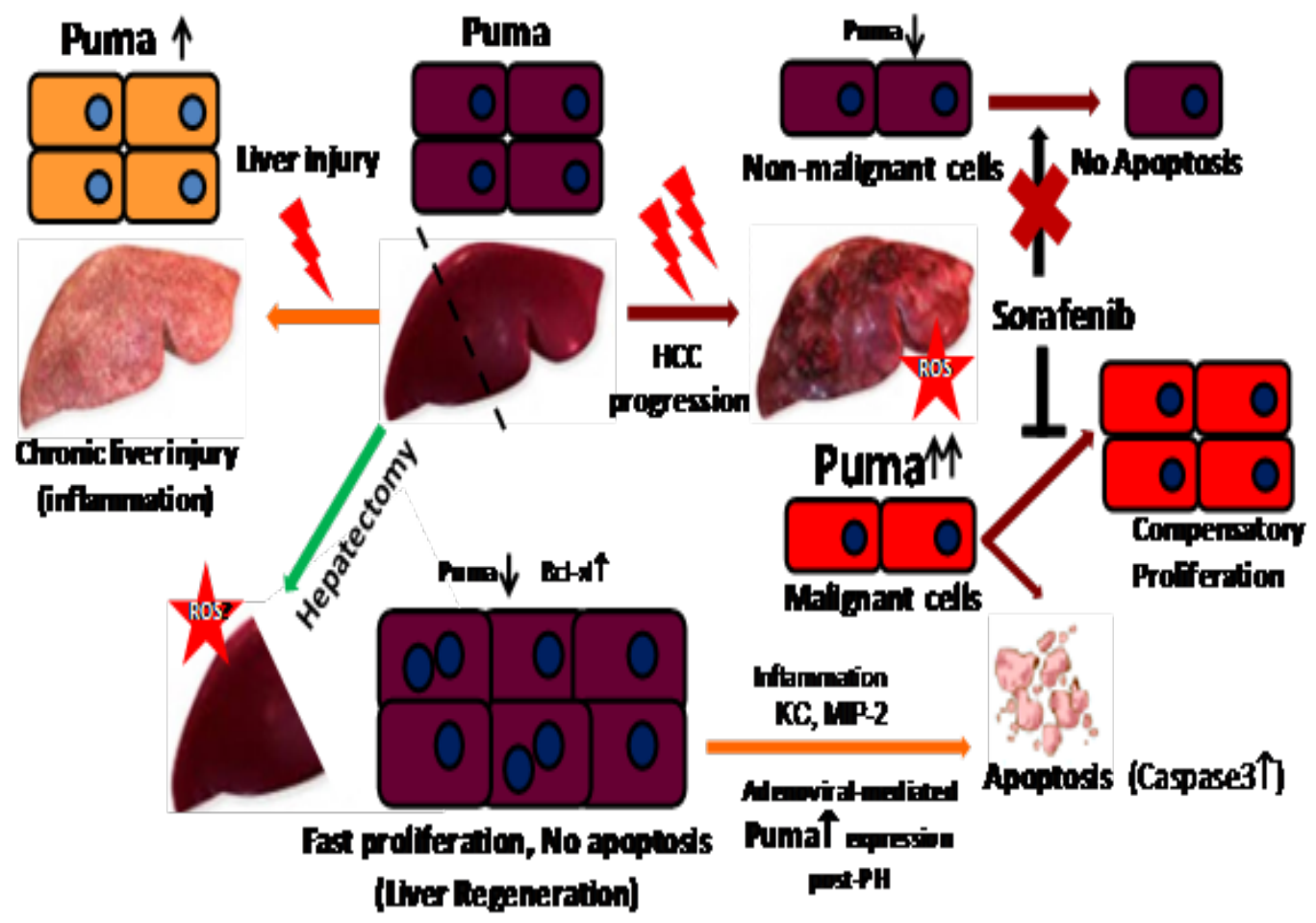

Figure I Context dependent Puma expression and regulation of hepatocyte fate during hepatocyte injury and regeneration.

Puma mediated apoptosis leads to hepatic injury and carcinogenesis

Apoptosis is the major mode of cell death in chronic viral hepatitis, though it is combined with necrosis (Necroptosis) in cholestatic livers. Multiple signaling pathways can activate an intermixed regulated apoptotic network by involving host, pathogen and microenvironment; including ER stress, death receptor-mediated cascade, ROS generation and mitochondrial dysfunction, whereas necrosis is an acute response. The patho physiologic role of apoptosis is complicated by contradictory evidences where lack of apoptosis or increased apoptosis may both result in carcinogenesis depending on the microenvironment and external influences. Our group and Qiu W et al. ${ }^{41}$ uncovered the long term controversy on the ambivalent role of the pro-apoptotic BH3 only protein Bid in tumorigenesis ${ }^{16,40-42}$ and Puma in carcinogen (i.e. DEN) driven liver cancer model of mice. ${ }^{16}$ Loss of $\mathrm{BH} 3$ only protein $\mathrm{Bid}^{43}$ or overexpression of $\mathrm{Bcl}-2^{44}$ suppressed DEN-induced liver cancer and hepatocyte proliferation. In mice deficient in NF-kB signaling ${ }^{45}$ or the anti-apoptotic Mcl$1^{46}$ developed spontaneous HCC secondary to hepatocyte death. Similarly Puma deficiency suppressed DEN induced compensatory proliferation in hepatocytes and tumor proliferation along with increased caspase 3 activation. Detailed signaling studies revealed that the JNK1-dependent Puma induction mediates DEN-induced hepatocyte apoptosis, proliferation and carcinogenesis. ${ }^{41}$ The acute, Puma-mediated apoptotic response in hepatocytes is a direct cause of compensatory proliferation and ensuing carcinogenesis. A twin role of Puma and apoptosis in cancer is rather unanticipated, but it is not without example. Puma deficiency was reported to defend hematopoietic stem/progenitor cells against irradiation induced cell death and suppresses compensatory proliferation and lymphomagenesis. ${ }^{17,18}$ Several genes can either promote or suppress tumorigenesis depending on the context. For example and as mentioned the inhibition of NF-kB enhanced DEN-induced HCC but reduced the colitis-induced colon cancer. ${ }^{47}$ Puma and apoptosis were induced by TNF- $\alpha$ or during inflammation through NF-kB. ${ }^{41,48}$ Interestingly, Puma-deficient HCCs or liver foci showed underlying proliferation long after the initial DEN abuse, but not significantly change in apoptosis. It may be possible that JNK1 mediated production of inflammatory cytokines support the induction of Puma, apoptosis and development of carcinogenesis beyond the initial acute injury phase. These findings provide additional mechanisms between chronic liver injury, inflammation and cancer that are consistent with higher levels of Puma in liver tumors compared with adjacent normal tissues in HCC patients. ${ }^{49}$

\section{Protective role of Puma in non-malignant cells: The therapeutic implication}

Next question come up with the implication of the Puma expression highlighting the therapeutic treatment in patients with HCC. Till date Sorafenib is the well-known approved drug for the treatment of patients with unresectable HCC. ${ }^{50,51}$ This multi kinase inhibitor targets serine/threonine kinases Raf/ERK as well as receptor tyrosine kinases VEGF receptor (VEGFR) and PDGF receptor (PDGFR) in HCC. ${ }^{52}$ Sorafenib reduces Mcl-1 expression, which is associated with the induction of apoptosis in some tumor cell lines. ${ }^{53,54}$ But till now the pro apoptotic properties of Sorafenib in normal hepatocyte are not 
clear. So in the therapeutic point of view it is important to evaluate the potential pro-apoptotic and anti-proliferative effect of sorafenib in healthy hepatocyte to understand its specific target on tumor cells. What is the effect of sorafenib on normal proliferation and liver regeneration? In fact, Sorafenib would not effect on non-malignant cells as well as regenerative cells. Liedtke group compared Sorafenibdependent signalling pathways in malignant hepatoma cells versus healthy hepatocytes in a syngeneic background and demonstrated that Sorafenib similarly reduced proliferation in hepatoma cells and in liver post $\mathrm{PH}$ whereas it particularly induces apoptosis only in malignant hepatoma cells in vivo and in vitro. ${ }^{55}$ Most interestingly as mentioned above also, the apoptosis induction in malignant cells was associated with the vigorous expression of Puma. In addition, Sorafenib reduced protein levels of the anti-apoptotic mediators such as Mcl-1, Bcl-2 and Bcl-xL, inhibited phosphorylation of the Akt target Mdm2 and endorsed strong activation of the caspase-3. Other studies indicated that in colon carcinoma cells Sorafenib-mediated apoptosis was associated with the induction of Puma. ${ }^{56}$ Sonntag $\mathrm{R}$ et al. ${ }^{55}$ measured Puma gene and protein expression in stimulated hepatoma cells, primary hepatocytes and Sorafenib-treated mice after $\mathrm{PH}$, respectively. Puma expression was significantly higher in hepatoma cells and even further induced after Sorafenib treatment when compared with hepatocytes. Immuno blot analysis revealed robust Puma protein expression in Hepa1-6 cells with or without Sorafenib treatment but clear decrease of Puma levels in Sorafenib treated primary hepatocytes. So the lower expression of Puma in normal hepatocytes is protecting cells from sorafenib induced cell death associated with Puma. After PH, Puma was drastically down regulated in Sorafenib-treated mice in both control and Sorafenibtreated groups. ${ }^{55}$ TShis also suggested that the normal proliferative and regenerative cells were not affected with sorafenib treatment due to low expression of Puma. The low expression of Puma protects the regenerative cells from sorafenib induced possibly Puma mediated apoptosis. Altogether, protection from Sorafenib driven apoptosis was found exclusively in non-malignant hepatocytes with low Puma levels in vitro and in vivo. Therefore the low expression of Puma in hepatocyte during normal proliferation process and liver regeneration protect them from sorafenib induced cell death. In contract the higher expression of Puma in malignant cells undergoes cell death with pro apoptotic and anti proliferative action of this therapeutic drug.

\section{Conclusion}

In normal proliferating processes such as liver regeneration the BH3 only protein Puma repression is necessary. Puma repression may contribute to the suppression of apoptosis and inflammation, thus promoting liver regeneration. The coupled reverse alterations of Puma and Bcl-xL may be a key cyto protective mechanism in response to oxidative stress stimuli after partial hepatectomy. In contrast during hepatitis and HCC the Puma expression increases with abnormal proliferating process, in agreement with above studies. These reverse results indicate that apoptosis is under strict control through the repression of Puma during the normal process, while the mis regulation of the apoptotic network may occur. Sorafenib can eliminate malignant hepatoma cells through apoptosis presumably in a Puma dependent manner.

\section{Acknowledgements}

None.

\section{Conflict of interest}

Author declares that there is no conflict of interest.

\section{References}

1. Adams JM, Cory S. The Bcl-2 apoptotic switch in cancer development and therapy. Oncogene. 2007;26(9):1324-1337.

2. Huang DC, Tschopp J, Strasser A. Bcl-2 does not inhibit cell death induced by the physiological Fas ligand: implications for the existence of type I and type II cells. Cell Death Differ. 2000;7(8):754-755.

3. Yu J, Zhang L, Hwang PM, et al. PUMA induces the rapid apoptosis of colorectal cancer cells. Mol Cell. 2001;7(3):673-682.

4. Nakano K, Vousden KH. PUMA, a novel proapoptotic gene, is induced by p53. Mol Cell. 2001;7(3):683-694.

5. Puthalakath H, Strasser A. Keeping killers on a tight leash: transcriptional and post-translational control of the pro-apoptotic activity of $\mathrm{BH}-$ 3-only proteins. Cell death and differentiation. 2002;9(5):505-512.

6. Wang X, Wang J, Lin S, et al. Sp1 is involved in $\mathrm{H}_{2} \mathrm{O}_{2}$-induced PUMA gene expression and apoptosis in colorectal cancer cells. J Exp Clin Cancer Res. 2008;27:44.

7. Steckley D, Karajgikar M, Dale LB, et al. Puma is a dominant regulator of oxidative stress induced Bax activation and neuronal apoptosis. $J$ Neurosci. 2007;27(47):12989-12999.

8. Han J, Flemington C, Houghton AB, et al. Expression of bbc3, a pro-apoptotic BH3-only gene, is regulated by diverse cell death and survival signals. Proc Natl Acad Sci U S A. 2001;98(20):11318-11323.

9. Ming L, Wang P, Bank A, et al. PUMA Dissociates Bax and Bcl$-\mathrm{X}(\mathrm{L})$ to induce apoptosis in colon cancer cells. $J$ Biol Chem. 2006;281(23):16034-16042.

10. Cartron PF, Gallenne T, Bougras G, et al. The first alpha helix of Bax plays a necessary role in its ligand-induced activation by the BH3-only proteins Bid and PUMA. Mol Cell. 2004;16(5):807-818.

11. Shibue T, Suzuki S, Okamoto H, et al. Differential contribution of Puma and Noxa in dual regulation of p53-mediated apoptotic pathways. EMBO J. 2006;25(20):4952-4962.

12. Li J, Lee B, Lee AS. Endoplasmic reticulum stress-induced apoptosis: multiple pathways and activation of p53-up-regulated modulator of apoptosis (PUMA) and NOXA by p53. J Biol Chem. 2006;281(11):7260-7270.

13. Yu J, Zhang L. PUMA, a potent killer with or without p53. Oncogene. 2008;27(Suppl 1):S71-S83.

14. Sun Q, Sakaida T, Yue W, et al. Chemo sensitization of head and neck cancer cells by PUMA. Mol Cancer Ther. 2007;6(12 Pt 1):3180-3188.

15. Hemann MT, Zilfou JT, Zhao Z, et al. Suppression of tumorigenesis by the p53 target PUMA. Proc Natl Acad Sci U S A. 2004;101(25):9333-9338.

16. Qiu W, Walter EBC, Kuan SF, et al. PUMA suppresses intestinal tumorigenesis in mice. Cancer Res. 2009;69(12):4999-5006.

17. Labi V, Erlacher M, Krumschnabel G, et al. Apoptosis of leukocytes triggered by acute DNA damage promotes lymphoma formation. Genes Dev. 2010;24(15):1602-1607.

18. Michalak EM, Vandenberg CJ, Delbridge AR, et al. Apoptosis-promoted tumorigenesis: gamma-irradiation-induced thymic lymphomagenesis requires Puma-driven leukocyte death. Genes Dev. 2010;24(15):1608-1613. 
19. Wirth TC, Manns MP. The impact of the revolution in hepatitis $C$ treat ment on hepatocellular carcinoma. Ann Oncol. 2016;27(8):1467-1474.

20. Adinolfi LE, Rinaldi L, Guerrera B, et al. NAFLD and NASH in HCV Infection: Prevalence and Significance in Hepatic and Extrahepatic Manifestations. Int J Mol Sci. 2016;17(6):E803.

21. Moreau R, Jalan R, Arroyo V. Acute-on-Chronic Liver Failure: Recent Concepts. J Clin Exp Hepatol. 2015;5(1):81-85.

22. Malhi H, Guicciardi ME, Gores GJ. Hepatocyte death: a clear and present danger. Physiol Rev. 2010;90(3):1165-1194.

23. Yamanaka N, Okamoto E, Kawamura E, et al. Dynamics of norma and injured human liver regeneration after hepatectomy as assessed on the basis of computed tomography and liver function. Hepatology. 1993;18(1):79-85.

24. Forbes SJ, Rosenthal N. Preparing the ground for tissue regeneration from mechanism to therapy. Nat Med. 2014;20(8):857-869.

25. Bird TG, Lorenzini S, Forbes SJ. Activation of stem cells in hepatic diseases. Cell Tissue Res. 2008;331(1):283-300.

26. Boulton RA, Alison MR, Golding M, et al. Augmentation of the early phase of liver regeneration after $70 \%$ partial hepatectomy in rats following selective Kupffer cell depletion. J Hepatol. 1998;29(2):271-280.

27. Meijer C, Wiezer MJ, Diehl AM, et al. Kupffer cell depletion by CI2MDP-liposomes alters hepatic cytokine expression and delays liver regeneration after partial hepatectomy. Liver. 2000;20(1):66-77.

28. Goh YP, Henderson NC, Heredia JE, et al. Eosinophils secrete IL-4 to facilitate liver regeneration. Proc Natl Acad Sci U S A. 2013;110(24):9914-9919.

29. Fan G, Kren BT, Steer CJ. Regulation of apoptosis-associated genes in the regenerating liver. Semin Liver Dis. 1998;18(2):123-140.

30. Lee FY, Li Y, Zhu H, et al. Tumor necrosis factor increases mitochondrial oxidant production and induces expression of uncoupling protein-2 in the regenerating mice [correction of rat] liver. Hepatology. 1999;29(3):677-687.

31. Tzung SP, Fausto N, Hockenbery DM. Expression of Bcl-2 family during liver regeneration and identification of Bcl-x as a delayed early response gene. Am J Pathol. 1997;150(6):1985-1995.

32. Kren BT, Trembley JH, Krajewski S, et al. Modulation of apoptosis-associated genes bcl-2, bcl-x, and bax during rat liver regeneration. Cell Growth Differ. 1996;7(12):1633-1642.

33. Rong Y, Distelhorst CW. Bcl-2 protein family members: versatile regulators of calcium signaling in cell survival and apoptosis. Annu Rev Physiol. 2008;70:73-91.

34. Li S, Zhao Y, He X, et al. Relief of extrinsic pathway inhibition by the Bid-dependent mitochondrial release of Smac in Fas-mediated hepatocyte apoptosis. J Biol Chem. 2002;277(30):26912-26920.

35. Taira K, Hiroyasu S, Shiraishi M, et al. Role of the Fas system in liver regeneration after a partial hepatectomy in rats. Eur Surg Res. 2001;33(5-6):334-341.

36. Chen S, Zheng J, Hao Q, et al. p53-insensitive PUMA down-regulation is essential in the early phase of liver regeneration after partial hepatectomy in mice. J Hepatol. 2010;52(6):864-871.

37. Wu WS, Heinrichs S, Xu D, et al. Slug antagonizes p53-mediated apoptosis of hematopoietic progenitors by repressing puma. Cell. 2005;123(4):641-653.

38. Eipel C, Schuett H, Glawe C, et al. Pifithrin-alpha induced p53 inhibition does not affect liver regeneration after partial hepatectomy in mice. J Hepatol. 2005;43(5):829-835.
39. Faouzi S, Burckhardt BE, Hanson JC, et al. Anti-Fas induces hepatic chemokines and promotes inflammation by an NF-kappa B-independent, caspase-3-dependent pathway. J Biol Chem. 2001;276(52):49077-49082.

40. Biswas S, Shi Q, Wernick A, et al. The loss of the BH3-only Bcl-2 family member Bid delays T-cell leukemogenesis in Atm-/-mice. Cell Death Differ. 2013;20(7):869-877.

41. Qiu W, Wang X, Leibowitz B, et al. PUMA-mediated apoptosis drives chemical hepato carcinogenesis in mice. Hepatology. 2011;54(4):1249-1258.

42. Egle A, Asslaber D, Villunger A, et al. Bid-ding for mercy: twisted killer in action. Cell Death Differ. 2013;20(7):847-849.

43. Bai L, Ni HM, Chen X, et al. Deletion of Bid impedes cell proliferation and hepatic carcinogenesis. Am J Pathol. 2005;166(5):1523-1532.

44. Pierce RH, Vail ME, Ralph L, et al. Bcl-2 expression inhibits liver carcinogenesis and delays the development of proliferating foci. Am J Pathol. 2002;160(5):1555-1560.

45. Luedde T, Beraza N, Kotsikoris V, et al. Deletion of NEMO/IKK gam$\mathrm{ma}$ in liver parenchymal cells causes steatohepatitis and hepatocellular carcinoma. Cancer Cell. 2007;11(2):119-132.

46. Weber T, Maas R, Auer J, et al. Arterial wave reflections and determinants of endothelial function a hypothesis based on peripheral mode of action. Am J Hypertens. 2007;20(3):256-262.

47. Greten FR, Eckmann L, Greten TF, et al. IKKbeta links inflammation and tumorigenesis in a mouse model of colitis-associated cancer. Cell. 2004;118(3):285-296.

48. Wang P, Qiu W, Dudgeon C, et al. PUMA is directly activated by NF-kappaB and contributes to TNF-alpha-induced apoptosis. Cell Death Differ. 2009;16(9):1192-1202.

49. Ahn $\mathrm{CH}$, Jeong EG, Kim SS, et al. Expressional and mutational analysis of pro-apoptotic Bcl-2 member PUMA in hepatocellular carcinomas. Dig Dis Sci. 2008;53(5):1395-1399.

50. Marra M, Sordelli IM, Lombardi A, et al. Molecular targets and oxidative stress biomarkers in hepatocellular carcinoma: an overview. J Transl Med. 2011;9:171

51. Montella L, Addeo R, Caraglia M, et al. Latest developments in targeted therapy for hepatocellular carcinoma. Expert Rev Anticancer Ther. 2010;10(10):1635-1646

52. Wilhelm SM, Adnane L, Newell P, et al. Preclinical overview of sorafenib, a multikinase inhibitor that targets both Raf and VEGF and PDGF receptor tyrosine kinase signaling. Mol Cancer Ther. 2008;7(10):3129-3140.

53. Liu L, Cao Y, Chen C, et al. Sorafenib blocks the RAF/MEK/ERK pathway, inhibits tumor angiogenesis, and induces tumor cell apoptosis in hepatocellular carcinoma model PLC/PRF/5. Cancer Res. 2006;66(24):11851-11858.

54. Yu C, Bruzek LM, Meng XW, et al. The role of Mcl-1 down regulation in the pro apoptotic activity of the multikinase inhibitor BAY 43-9006. Oncogene. 2005;24(46):6861-6869.

55. Sonntag R, Gassler N, Bangen JM, et al. Pro-apoptotic Sorafenib signaling in murine hepatocytes depends on malignancy and is associated with PUMA expression in vitro and in vivo. Cell Death Dis. 2014;5:e1030.

56. Dudgeon $\mathrm{C}$, Peng R, Wang P, et al. Inhibiting oncogenic signaling by sorafenib activates PUMA via GSK3beta and NF-kappaB to suppress tumor cell growth. Oncogene. 2012;31(46):4848-4858. 